Journal of Nonlinear Mathematical Physics

ISSN (Online): 1402-9251 ISSN (Print): 1776-0852

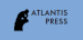
Journal Home Page: https://www.atlantis-press.com/journals/jnmp

The modified Korteweg-de Vries equation on the quarter plane with $t$-periodic data

Guenbo Hwang

To cite this article: Guenbo Hwang (2017) The modified Korteweg-de Vries equation on the quarter plane with $t$-periodic data, Journal of Nonlinear Mathematical Physics 24:4, 620-634, DOI: https://doi.org/10.1080/14029251.2017.1375695

To link to this article: https://doi.org/10.1080/14029251.2017.1375695

Published online: 04 January 2021 


\title{
The modified Korteweg-de Vries equation on the quarter plane with $t$-periodic data
}

\author{
Guenbo Hwang \\ Department of Mathematics and Institute of Basic Science, Daegu University, \\ Gyeongsan Gyeongbuk 38453, Korea \\ ghwang@daegu.ac.kr
}

Received 20 June 2017

Accepted 17 July 2017

\begin{abstract}
We study the modified Korteweg-de Vries equation posed on the quarter plane with asymptotically $t$-periodic Dirichlet boundary datum $u(0, t)$ in the sense that $u(0, t)$ tends to a periodic function $\tilde{g}_{0}(t)$ with period $\tau$ as $t \rightarrow \infty$. We consider the perturbative expansion of the solution in a small $\varepsilon>0$. Here we show that if the unknown boundary data $u_{x}(0, t)$ and $u_{x x}(0, t)$ are asymptotically $t$-periodic with period $\tau$ which tend to the functions $\tilde{g}_{1}(t)$ and $\tilde{g}_{2}(t)$ as $t \rightarrow \infty$, respectively, then the periodic functions $\tilde{g}_{1}(t)$ and $\tilde{g}_{2}(t)$ can be uniquely determined in terms of the function $\tilde{g}_{0}(t)$. Furthermore, we characterize the Fourier coefficients of $\tilde{g}_{1}(t)$ and $\tilde{g}_{2}(t)$ to all orders in the perturbative expansion by solving an infinite system of algebraic equations. As an illustrative example, we consider the case of a sine-wave as Dirichlet datum and we explicitly determine the coefficients for large $t$ up to the third order in the perturbative expansion.
\end{abstract}

Keywords: Initial-boundary value problem; Integrable systems; Modified Korteweg-de Vries equation.

2000 Mathematics Subject Classification: 37K15, 35Q15

\section{Introduction}

A unified method, also known as the Fokas method, was introduced in [1-3] and extensively applied for analyzing boundary value problems (see also the monograph [4] and references therein). The method yields a novel integral representation of the solution in terms of the unique solution of a matrix Riemann-Hilbert problem whose jump matrices are formulated by the so-called spectral functions. These spectral functions are defined by the initial value and all boundary values. The most difficult step in the implementation of the method is the characterization of the unknown boundary values, known as the generalized Dirichlet-to-Neumann map [5]. For example, for the Dirichlet problem of the modified Korteweg-de Vries equation $(\mathrm{mKdV})$ posed on the half-line [6], the initial $u(x, 0)$ and Dirichlet boundary $u(0, t)$ conditions are given, while boundary conditions $u_{x}(0, t)$ and $u_{x x}(0, t)$ are unknown in view of the well-posedness. Hence in the implementation of the method, it is necessary that these unknown boundary values should be characterized in terms of the known initial and boundary data. For the case of the vanishing boundary conditions for large $t$, due to the explicit exponential $(x, t)$-dependence in the representation of the solution, it is possible to characterize the unknown boundary values by using the Deift-Zhou and the Deift-Zhou-Venakides methods for the asymptotic analysis of the Riemann-Hilbert problem [7-10]. However, for the case that the boundary data are periodic in $t$, it require the explicit characterization of the unknown boundary values [11-13]. In a recent development of this characterization, a new perturbative approach was proposed in [17] (see also [6,14-16]). For example, it was shown in [6] for the mKdV on the halfline that if $u(0, t)=\varepsilon \sin t$ with $\varepsilon>0$, then the boundary data $u_{x}(0, t)$ and $u_{x x}(0, t)$ can be computed 
up to the third order of $\varepsilon$ in the perturbative expansion and these functions are also periodic as $t \rightarrow \infty$ up to this third order. However, since the computations for the coefficients of $u_{x}(0, t)$ and $u_{x x}(0, t)$ in the perturbative expansion involve rather complicated integrals and it is quite difficult to go beyond higher order terms.

In this paper, we consider the $\mathrm{mKdV}$ equation posed on the quarter plane

$$
u_{t}+u_{x x x}-6 \lambda u^{2} u_{x}=0, \quad x>0, \quad t>0,
$$

where $\lambda= \pm 1$ and $u(x, t)$ decays rapidly for all $t$ as $x \rightarrow \infty$. We assume that the given initial condition $u_{0}(x)=u(x, 0)$ vanishes sufficiently fast as $x \rightarrow \infty$. We denote the given boundary datum $u(0, t)$ and the unknown boundary data $u_{x}(0, t)$ and $u_{x x}(0, t)$ as

$$
g_{0}(t)=u(0, t), \quad g_{1}(t)=u_{x}(0, t), \quad g_{2}(t)=u_{x x}(0, t),
$$

which are assumed to be sufficiently smooth. Moreover, we assume that the boundary data are asymptotically periodic as $t \rightarrow \infty$ in the sense that

$$
g_{j}(t)=\tilde{g}_{j}(t)+O\left(t^{-7 / 2}\right), \quad t \rightarrow \infty, \quad j=0,1,2,
$$

where $\tilde{g}_{j}(t), j=0,1,2$, are periodic functions with period $\tau>0$. Here, we consider the perturbative expansion of the solution for (1.1) in a small $\varepsilon>0$ and we characterize the Dirichlet-to-Neumann map for the unknown boundary data in the limit of large $t$ and small $\varepsilon>0$. More specifically, we will show that if the boundary data are asymptotically periodic as $t \rightarrow \infty$, the functions $\tilde{g}_{1}(t)$ and $\tilde{g}_{2}(t)$ can be uniquely determined by solving an infinite system of algebraic equations. In contrast to the case of the nonlinear problem, the result of the linearized equation obtained from (1.1) by deleting the nonlinear term is remarkable. It can be shown that if the known boundary datum $g_{0}(t)$ is asymptotically periodic as $t \rightarrow \infty$, then so are the unknown boundary data $g_{1}(t)$ and $g_{2}(t)$. It should be noted that the approach in this paper is based on the method introduced for the nonlinear Schrödinger equation in [18].

As an illustrative example, we consider the case of a sine-wave as Dirichlet datum

$$
g_{0}(t)=\varepsilon \sin t, \quad \varepsilon>0, \quad t>0 .
$$

It was proved in [6] that up to the third order in the perturbtaive expansion, the unknown boundary data $g_{1}(t)$ and $g_{2}(t)$ are asymptotically periodic with the same period as $g_{0}(t)$. However, the expressions at $O\left(\varepsilon^{3}\right)$ involve rather complicated integrals. Indeed, the perturbative approach presented in [6] is quite difficult to find the coefficients for the higher order terms. In this paper, we can explicitly determine the coefficients for the third order term by using the presented perturbative approach. This perturbative approach is much easier than the one presented in [6] and it is possible to find coefficients for the higher order terms. However, it should be noted that the perturbative approach in [6] does not require the assumption that the unknown boundary data are asymptotically periodic as $t \rightarrow \infty$.

\section{The linearized $\mathrm{mKdV}$ equation}

In this section, first we study the linearized $\mathrm{mKdV}$ equation also known as linear KdV equation

$$
u_{t}+u_{x x x}=0, \quad x>0, \quad t>0 .
$$


The equation admits an overdetermined linear system called Lax pair

$$
\begin{aligned}
& \varphi_{x}+i f_{1}(k) \varphi=u, \\
& \varphi_{t}+i f_{2}(k) \varphi=4 k^{2} u+2 i k u_{x}-u_{x x},
\end{aligned}
$$

where

$$
f_{1}(k)=2 k, \quad f_{2}(k)=8 k^{3} .
$$

The Lax pair (2.2) can be written as

$$
d\left(e^{i f_{1}(k) x+i f_{2}(k) t} \varphi\right)=W(x, t, k),
$$

where the closed differential 1-form $W(x, t, k)$ is given by

$$
W(x, t, k)=e^{i f_{1}(k) x+i f_{2}(k) t}\left[u d x+\left(4 k^{2} u+2 i k u_{x}-u_{x x}\right) d t\right] .
$$

We then introduce the domains $D_{+}=\left\{k \in \mathbb{C} \mid \operatorname{Im} f_{2}(k)>0\right\}$ and it is convenient to decompose $D_{+}$ as $D_{+}=D_{+, 1} \cup D_{+, 2} \cup D_{+, 3}$ (see fig. 1), where

$$
\begin{aligned}
& D_{+, 1}=\{k \in \mathbb{C} \mid 0<\arg k<\pi / 3\}, \\
& D_{+, 2}=\{k \in \mathbb{C} \mid 2 \pi / 3<\arg k<\pi\}, \\
& D_{+, 3}=\{k \in \mathbb{C} \mid 4 \pi / 3<\arg k<5 \pi / 3\} .
\end{aligned}
$$

Integrating (2.3) along $(0, \infty) \times(0, t)$ and using the Green theorem, we find the following global relation for the linearized $\mathrm{mKdV}$ equation

$$
\hat{u}_{0}(k)-e^{i f_{2}(k) t} \hat{u}(k, t)+\hat{G}(k, t)=0, \quad \operatorname{Im} k \geq 0,
$$

where

$$
\begin{aligned}
\hat{u}(k, t) & =\int_{0}^{\infty} e^{i f_{1}(k) x} u(x, t) d x, \quad \operatorname{Im} k \geq 0, \\
\hat{G}(k, t) & =-4 k^{2} \hat{g}_{0}(k, t)-2 i k \hat{g}_{1}(k, t)+\hat{g}_{2}(k, t)
\end{aligned}
$$

with

$$
\hat{g}_{j}(k, t)=\int_{0}^{t} e^{i f_{2}(k) s} g_{j}(s) d s, \quad j=0,1,2 .
$$

Multiplying (2.4) by $\frac{1}{\pi} e^{-i f_{1}(k) x-i f_{2}(k) t}$ and taking the inverse Fourier transform, we find

$$
u(x, t)=\frac{1}{\pi} \int_{-\infty}^{\infty} e^{-i f_{1}(k) x-i f_{2}(k) t} \hat{u}_{0}(k) d k+\frac{1}{\pi} \int_{-\infty}^{\infty} e^{-i f_{1}(k) x-i f_{2}(k) t} \hat{G}(k, t) d k .
$$

Deforming the contour $(-\infty, \infty)$ to $\partial D_{+, 3}$ for the second integral in (2.5), the solution of (2.1) is given by

$$
u(x, t)=\frac{1}{\pi} \int_{-\infty}^{\infty} e^{-i f_{1}(k) x-i f_{2}(k) t} \hat{u}_{0}(k) d k-\frac{1}{\pi} \int_{\partial D_{+, 3}} e^{-i f_{1}(k) x-i f_{2}(k) t} \hat{G}(k, t) d k .
$$

Note that the solution (2.6) depends on the known and unknown boundary data $u(0, t), u_{x}(0, t)$ and $u_{x x}(0, t)$ via $\hat{g}_{j}(k, t), j=0,1,2$, in the definition of the function $\hat{G}(k, t)$. However, the global relation (2.4) can be used to determine the unknown boundary data. 


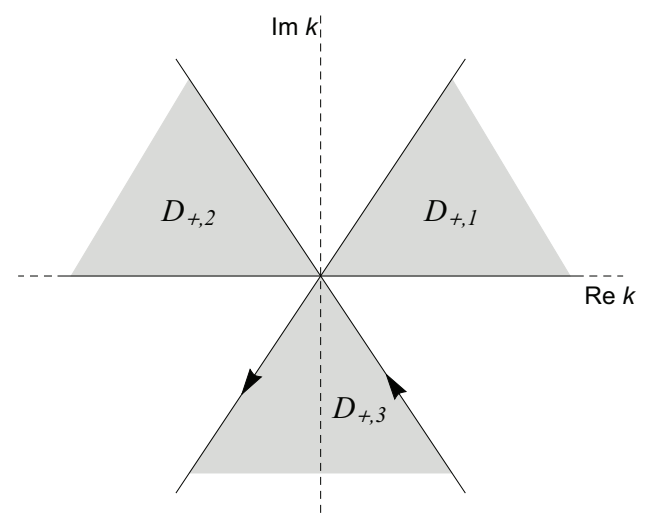

Fig. 1. The region $D_{+}$(shaded) of the complex $k$-plane where $\operatorname{Im} f_{2}(k)>0$ with $f_{2}(k)=8 k^{3}$.

The transformation $k \rightarrow \alpha(k)$ leaves $f_{2}(k)$ invariant if $k^{3}-\alpha^{3}(k)=0$ and hence $\hat{g}_{j}(k, t)$ is invariant under this transformation. The equation $k^{3}-\alpha^{3}(k)=0$ has two nontrivial roots, namely, $\alpha(k)=\alpha k, \alpha^{2} k$, where $\alpha=e^{2 i \pi / 3}$. Letting $k \rightarrow \alpha k$ and $k \rightarrow \alpha^{2} k$ in (2.4), we find the following equations for $k \in D_{+, 3}$

$$
\begin{aligned}
& \hat{u}_{0}(\alpha k)-e^{i f_{2}(k) t} \hat{u}(\alpha k, t)=4 \alpha^{2} k^{2} \hat{g}_{0}(k, t)+2 i \alpha k \hat{g}_{1}(k, t)-\hat{g}_{2}(k, t), \\
& \hat{u}_{0}\left(\alpha^{2} k\right)-e^{i f_{2}(k) t} \hat{u}\left(\alpha^{2} k, t\right)=4 \alpha k^{2} \hat{g}_{0}(k, t)+2 i \alpha^{2} k \hat{g}_{1}(k, t)-\hat{g}_{2}(k, t) .
\end{aligned}
$$

Solving the linear system (2.7) for $\hat{g}_{1}$ and $\hat{g}_{2}$, we find

$$
\begin{aligned}
\hat{g}_{1}(k, t)= & -2 i k \hat{g}_{0}(t)-\frac{\sqrt{3}}{6 k}\left[\hat{u}_{0}(\alpha k)-\hat{u}_{0}\left(\alpha^{2} k\right)-e^{i f_{2}(k) t}\left(\hat{u}(\alpha k, t)-\hat{u}\left(\alpha^{2} k, t\right)\right)\right], \\
\hat{g}_{2}(k, t)= & -4 k^{2} \hat{g}_{0}(k)-\frac{i}{\sqrt{3}}\left[\alpha^{-1} \hat{u}_{0}(\alpha k)-\alpha^{-2} \hat{u}_{0}\left(\alpha^{2} k\right)\right. \\
& \left.-e^{i f_{2}(k) t}\left(\alpha^{-1} \hat{u}(\alpha k, t)-\alpha^{-2} \hat{u}\left(\alpha^{2} k, t\right)\right)\right] .
\end{aligned}
$$

Substituting (2.8) into (2.6), the solution of (2.1) is given by

$$
\begin{aligned}
u(x, t)= & \frac{1}{\pi} \int_{-\infty}^{\infty} e^{-i f_{1}(k) x-i f_{2}(k) t} \hat{u}_{0}(k) d k+\frac{12}{\pi} \int_{\partial D_{+, 3}} k^{2} e^{-i f_{1}(k) x-i f_{2}(k) t} \hat{g}_{0}(k, t) d k \\
& -\frac{1}{\pi} \int_{\partial D_{+, 3}} e^{-i f_{1}(k) x-i f_{2}(k) t}\left(\alpha \hat{u}_{0}(\alpha k)+\alpha^{2} \hat{u}_{0}\left(\alpha^{2} k\right)\right) d k,
\end{aligned}
$$

where we have used the fact that the terms involving $e^{i f_{2}(k) t}$ in (2.8) do not contribute to (2.6) thanks to the Cauchy theorem. Also, we deform the contour $\partial D_{+, 3}$ to $(-\infty, \infty)$ in the third integral of (2.9), which leads to

$$
\begin{aligned}
u(x, t)= & \frac{1}{\pi} \int_{-\infty}^{\infty} e^{-i f_{1}(k) x-i f_{2}(k) t}\left(\hat{u}_{0}(k)+\alpha \hat{u}_{0}(\alpha k)+\alpha^{2} \hat{u}_{0}\left(\alpha^{2} k\right)\right) d k \\
& +\frac{12}{\pi} \int_{\partial D_{+, 3}} k^{2} e^{-i f_{1}(k) x-i f_{2}(k) t} \hat{g}_{0}(k, t) d k
\end{aligned}
$$


Note that by integration by parts, the function $\hat{g}_{0}(k, t)$ can be written as

$$
\hat{g}_{0}(k, t)=\frac{1}{i f_{2}(k)}\left(e^{i f_{2}(k) t} g_{0}(t)-g_{0}(0)\right)-\frac{1}{i f_{2}(k)} \int_{0}^{t} e^{i f_{2}(k) s} \dot{g}_{0}(s) d s .
$$

Using the above equation and deforming the contour $\partial D_{+, 3}$ to pass to the right of the singularity $k=0$, which is denoted by $\partial D_{+.3}^{0}$, equation (2.10) yields

$$
\begin{aligned}
u(x, t)= & \frac{1}{\pi} \int_{-\infty}^{\infty} e^{-i f_{1}(k) x-i f_{2}(k) t}\left(\hat{u}_{0}(k)+\alpha \hat{u}_{0}(\alpha k)+\alpha^{2} \hat{u}_{0}\left(\alpha^{2} k\right)\right) d k+g_{0}(t) \\
& -\frac{3}{2 i \pi} \int_{\partial D_{+, 3}^{0}} \frac{1}{k} e^{-i f_{1}(k) x-i f_{2}(k) t}\left(g_{0}(0)+\int_{0}^{t} e^{i f_{2}(k) s} \dot{g}_{0}(s) d s\right) d k .
\end{aligned}
$$

Differentiating (2.11) with respect to $x$ and evaluating at $x=0$, we find the unknown boundary value $g_{1}(t)$ given by

$$
\begin{aligned}
g_{1}(t)= & \frac{2}{i \pi} \int_{-\infty}^{\infty} k e^{-i f_{2}(k) t}\left(\hat{u}_{0}(k)+\alpha \hat{u}_{0}(\alpha k)+\alpha^{2} \hat{u}_{0}\left(\alpha^{2} k\right)\right) d k \\
& +\frac{3}{\pi} \int_{\partial D_{+, 3}} e^{-i f_{2}(k) t}\left(g_{0}(0)+\int_{0}^{t} e^{i f_{2}(k) s} \dot{g}_{0}(s) d s\right) d k .
\end{aligned}
$$

Using the following equation,

$$
\int_{\partial D_{+, 3}} e^{-i f_{2}(k) t} d k=\frac{\alpha_{1}}{t^{1 / 3}}, \quad \alpha_{1}=-\frac{\sqrt{3}}{6} \Gamma(1 / 3),
$$

equation (2.12) is equivalent to

$$
\begin{aligned}
g_{1}(t)= & \frac{2}{i \pi} \int_{-\infty}^{\infty} k e^{-i f_{2}(k) t}\left(\hat{u}_{0}(k)+\alpha \hat{u}_{0}(\alpha k)+\alpha^{2} \hat{u}_{0}\left(\alpha^{2} k\right)\right) d k \\
& +\frac{3 \alpha_{1}}{\pi}\left(\frac{g_{0}(0)}{t^{1 / 3}}+\int_{0}^{t} \frac{\dot{g}_{0}(s)}{(t-s)^{1 / 3}} d s\right) .
\end{aligned}
$$

Similarly, differentiating (2.11) twice with respect to $x$ and evaluating at $x=0$, we find the unknown boundary value $g_{2}(t)$ given by

$$
\begin{aligned}
g_{2}(t)= & -\frac{4}{\pi} \int_{-\infty}^{\infty} k^{2} e^{-i f_{2}(k) t}\left(\hat{u}_{0}(k)+\alpha \hat{u}_{0}(\alpha k)+\alpha^{2} \hat{u}_{0}\left(\alpha^{2} k\right)\right) d k \\
& +\frac{6}{i \pi} \int_{\partial D_{+, 3}} k e^{-i f_{2}(k) t}\left(g_{0}(0)+\int_{0}^{t} e^{i f_{2}(k) s} \dot{g}_{0}(s) d s\right) d k
\end{aligned}
$$

Also note that using the following equation

$$
\int_{\partial D_{+, 3}} k e^{-i f_{2}(k) t} d k=\frac{\alpha_{2}}{t^{2 / 3}}, \quad \alpha_{2}=-\frac{i \sqrt{3}}{12} \Gamma(2 / 3),
$$

equation (2.14) is also equivalent to

$$
\begin{aligned}
g_{2}(t)= & -\frac{4}{\pi} \int_{-\infty}^{\infty} k^{2} e^{-i f_{2}(k) t}\left(\hat{u}_{0}(k)+\alpha \hat{u}_{0}(\alpha k)+\alpha^{2} \hat{u}_{0}\left(\alpha^{2} k\right)\right) d k \\
& +\frac{6 \alpha_{2}}{i \pi}\left(\frac{g_{0}(0)}{t^{2 / 3}}+\int_{0}^{t} \frac{\dot{g}_{0}(s)}{(t-s)^{2 / 3}} d s\right) .
\end{aligned}
$$


G. Hwang / The modified Korteweg-de Vries equation on the quarter plane with t-periodic data

Proposition 2.1. Assume that $\tilde{g}_{0}(t)$ is a smooth periodic function with period $\tau=2 \pi / \omega>0$ and has the Fourier series

$$
\tilde{g}_{0}(t)=\sum_{n=-\infty}^{\infty} a_{n} e^{i n \omega t}, \quad t \geq 0
$$

where $a_{n} \in \mathbb{C}$ are the Fourier coefficients and $a_{0}=0$. Then, there are unique periodic functions $\tilde{g}_{1}(t)$ and $\tilde{g}_{2}(t)$ such that $u(0, \cdot)-\tilde{g}_{0}$ is in the Schwartz class, denoted by $\in \mathscr{S}[0, \infty)$ and

$$
u_{x}(0, t)-\tilde{g}_{1}(t)=O\left(t^{-1 / 3}\right), \quad u_{x x}(0, t)-\tilde{g}_{2}(t)=O\left(t^{-2 / 3}\right), \quad t \rightarrow \infty .
$$

Moreover, the functions $\tilde{g}_{1}(t)$ and $\tilde{g}_{2}(t)$ have period $\tau$ and the Fourier series are given by

$$
\tilde{g}_{1}(t)=\sum_{n=-\infty}^{\infty} b_{n} e^{i n \omega t}, \quad b_{n}= \begin{cases}-i e^{-i \pi / 3}(n \omega)^{1 / 3} a_{n}, & n \geq 0, \\ -i e^{-2 i \pi / 3}(-n \omega)^{1 / 3} a_{n}, & n<0\end{cases}
$$

and

$$
\tilde{g}_{2}(t)=\sum_{n=-\infty}^{\infty} c_{n} e^{i n \omega t}, \quad c_{n}= \begin{cases}-e^{-2 i \pi / 3}(n \omega)^{2 / 3} a_{n}, & n \geq 0, \\ -e^{-4 i \pi / 3}(-n \omega)^{2 / 3} a_{n}, & n<0,\end{cases}
$$

where $s^{1 / 3}$ is the principal value of the third root of $s>0$.

Proof. Let $u(x, t)$ be the solution given in (2.11) with $g_{0}(t)=\tilde{g}_{0}(t)$. We first prove that $g_{1}(t)-$ $\tilde{g}_{1}(t)=O\left(t^{-1 / 3}\right)$ as $t \rightarrow \infty$. From (2.12), we write $g_{1}(t)=I_{1}(t)+I_{2}(t)$, where

$$
\begin{aligned}
& I_{1}(t)=\frac{2}{i \pi} \int_{-\infty}^{\infty} k e^{-i f_{2}(k) t}\left(\hat{u}_{0}(k)+\alpha \hat{u}_{0}(\alpha k)+\alpha^{2} \hat{u}_{0}\left(\alpha^{2} k\right)\right) d k, \\
& I_{2}(t)=\frac{3}{\pi} \int_{\partial D_{+, 3}} e^{-i f_{2}(k) t}\left(g_{0}(0)+\int_{0}^{t} e^{i f_{2}(k) s} \dot{g}_{0}(s) d s\right) d k .
\end{aligned}
$$

We will show that $I_{1}(t)=O\left(t^{-1 / 3}\right)$ as $t \rightarrow \infty$. Indeed, using integration by parts and deforming the contour $(-\infty, \infty)$ to $\partial D_{+, 3}$, note that

$$
\int_{-\infty}^{\infty} k e^{-i f_{2}(k) t} \hat{u}_{0}(k) d k=\frac{\alpha_{1} u_{0}(0)}{2 i t^{1 / 3}}+\frac{1}{2 i} \int_{0}^{\infty} \dot{u}_{0}(x) \int_{\partial D_{+, 3}} e^{-i f_{2}(k) t+2 i k x} d k d x .
$$

For the integral $\int_{\partial D_{+, 3}} e^{-i f_{2}(k) t+2 i k x} d k$ in (2.21), deforming the contour $\partial D_{+, 3}$ to the contour $\left\{r e^{-i \pi / 6} \mid r \geq 0\right\}$ and using the Laplace method for asymptotic evaluation of integrals, we find

$$
\int_{\partial D_{+, 3}} e^{-i f_{2}(k) t+2 i k x} d k \sim-\frac{e^{-i \pi / 6}}{2}(3+i \sqrt{3}) \int_{0}^{\delta} e^{-8 r^{3} t} d r
$$

for small $\delta>0$. Evaluating the rhs of (2.22), we find

$$
\int_{\partial D_{+, 3}} e^{-i f_{2}(k) t+2 i k x} d k=-\frac{e^{-i \pi / 6}}{12 t^{1 / 3}}(3+i \sqrt{3}) \Gamma(1 / 3)+O\left(t^{-2 / 3}\right), \quad t \rightarrow \infty,
$$

which implies that

$$
\int_{-\infty}^{\infty} k e^{-i f_{2}(k) t} \hat{u}_{0}(k) d k=O\left(t^{-1 / 3}\right), \quad t \rightarrow \infty .
$$

We can evaluate the rest of the integrals in (2.20a) in a similar way and then we prove that $I_{1}(t)=$ $O\left(t^{-1 / 3}\right)$ as $t \rightarrow \infty$. 
Note that by integration by parts in $(2.20 \mathrm{~b})$, the integral $I_{2}(t)$ can be written as

$$
I_{2}(t)=\frac{1}{\pi} \int_{\partial D_{+, 3}}\left(\frac{k f_{2}^{\prime}(k)}{f_{2}(k)} g_{0}(t)-i k f_{2}^{\prime}(k) \int_{0}^{t} e^{-i(t-s) f_{2}(k)} g_{0}(s) d s\right) d k .
$$

Substituting the Fourier expansion (2.16) into the above equation, we find

$$
\begin{aligned}
I_{2}(t) & =\frac{1}{\pi} \int_{\partial D_{+, 3}} k f_{2}^{\prime}(k) \sum_{n \neq 0} a_{n}\left(\frac{e^{i n \omega t}}{f_{2}(k)}-i \int_{0}^{t} e^{-i(t-s) f_{2}(k)+i n \omega s} d s\right) d k \\
& =\frac{1}{\pi} \int_{\partial D_{+, 3}} k f_{2}^{\prime}(k) \sum_{n \neq 0} a_{n}\left(\frac{e^{i n \omega t}}{f_{2}(k)}-\frac{e^{i n \omega t}-e^{-i f_{2}(k) t}}{f_{2}(k)+n \omega}\right) d k .
\end{aligned}
$$

For each $n \in \mathbb{Z}$, let $k_{n}$ be a root of the equation $f_{2}(k)+n \omega=0$ in $\partial D_{+, 3}$, where $k_{n}$ is given by

$$
k_{n}= \begin{cases}\frac{1}{2} e^{-i \pi / 3}(n \omega)^{1 / 3}, & n \geq 0, \\ \frac{1}{2} e^{-2 i \pi / 3}(-n \omega)^{1 / 3}, & n<0 .\end{cases}
$$

We deform the contour $\partial D_{+, 3}$ to passe to the right of the singularites $k=k_{n}$, which is denoted by $\partial \hat{D}_{+, 3}$, so that we can split the integral (2.23) as

$$
\begin{aligned}
I_{2}(t)= & \frac{1}{\pi} \sum_{n \neq 0} a_{n} e^{i n \omega t} \int_{\partial \hat{D}_{+, 3}} k f_{2}^{\prime}(k)\left(\frac{1}{f_{2}(k)}-\frac{1}{f_{2}(k)+n \omega}\right) d k \\
& +\frac{1}{\pi} \int_{\partial \hat{D}_{+, 3}} k f_{2}^{\prime}(k) e^{-i f_{2}(k) t} \sum_{n \neq 0}\left(\frac{a_{n}}{f_{2}(k)+n \omega}\right) d k .
\end{aligned}
$$

The first integral in (2.24) can be evaluated by the Residue theorem

$$
\frac{1}{\pi} \sum_{n \neq 0} a_{n} e^{i n \omega t} \int_{\partial \hat{D}_{+, 3}} k f_{2}^{\prime}(k)\left(\frac{1}{f_{2}(k)}-\frac{1}{f_{2}(k)+n \omega}\right) d k=-2 i \sum_{n \neq 0} a_{n} e^{i n \omega t} k_{n}=\tilde{g}_{1}(t) .
$$

Regarding the second integral in (2.24), we deform $\partial \hat{D}_{+, 3}$ to the steepest descent contour $\Gamma=$ $\left\{r e^{-i \pi / 6} \mid r \geq 0\right\} \cup\left\{r e^{5 i \pi / 6} \mid r \geq 0\right\}$ and then the steepest descent method yields

$$
\frac{1}{\pi} \int_{\partial \hat{D}_{+, 3}} k f_{2}^{\prime}(k) e^{-i f_{2}(k) t} \sum_{n \neq 0}\left(\frac{a_{n}}{f_{2}(k)+n \omega}\right) d k=O\left(t^{-4 / 3}\right), \quad t \rightarrow \infty
$$

Thus, $g_{1}(t)=\tilde{g}_{1}(t)+O\left(t^{-1 / 3}\right)$ as $t \rightarrow \infty$.

In a similar argument, we can also prove that $g_{2}(t)=\tilde{g}_{2}(t)+O\left(t^{-2 / 3}\right)$ as $t \rightarrow \infty$ where $g_{2}(t)$ and $\tilde{g}_{2}(t)$ are given by (2.14) and (2.19), respectively. Also, the uniqueness of $\tilde{g}_{1}(t)$ and $\tilde{g}_{2}(t)$ follows from the fact that $u(0, \cdot)-\tilde{g}_{0}(t) \in \mathscr{S}[0, \infty)$ as discussed in [18]. 
G. Hwang / The modified Korteweg-de Vries equation on the quarter plane with t-periodic data

\section{The modified KdV equation}

\subsection{Eigenfunctions}

We now consider the modified $\mathrm{KdV}$ equation posed on the quarter plane

$$
u_{t}+u_{x x x}-6 \lambda u^{2} u_{x}=0, \quad x>0, \quad t>0,
$$

where $\lambda= \pm 1$. We assume that $u_{0}(x)=u(x, 0)$ and $g_{0}(t)=u(0, t)$ are given, while $g_{1}(t)=u_{x}(0, t)$ and $g_{2}(t)=u_{x x}(0, t)$ are unknown. It is known that (3.1) admits the following Lax pair

$$
\begin{aligned}
\mu_{x}+i f_{1}(k) \hat{\sigma}_{3} \mu & =U(x, t) \mu, \\
\mu_{t}+i f_{2}(k) \hat{\sigma}_{3} \mu & =V(x, t, k) \mu,
\end{aligned}
$$

where $k \in \mathbb{C}$ is a spectral parameter, $\mu(x, t, k)$ is a $2 \times 2$ matrix-valued eigenfunction, $\hat{\sigma}_{3} A=\left[\sigma_{3}, A\right]$ with $\sigma_{3}=\operatorname{diag}(1,-1)$ and

$$
\begin{aligned}
& f_{1}(k)=2 k, \quad f_{2}(k)=8 k^{3}, \quad U(x, t)=\left(\begin{array}{cc}
0 & u \\
\lambda u & 0
\end{array}\right), \\
& V(x, t, k)=2 U^{3}-U_{x x}-2 i k\left(U^{2}+U_{x}\right) \sigma_{3}+4 k^{2} U .
\end{aligned}
$$

We define the normalized solutions of (3.2) at $\left(x_{2}, t_{2}\right)=(0,0)$ and $\left(x_{3}, t_{3}\right)=(\infty, t)$ in terms of the linear Volterra integral equations

$$
\mu_{j}(x, t, k)=I+\int_{\left(x_{j}, t_{j}\right)}^{(x, t)} e^{i\left(f_{1}(k)\left(x^{\prime}-x\right)+f_{2}(k)\left(t^{\prime}-t\right)\right) \hat{\sigma}_{3}} W_{j}\left(x^{\prime}, t^{\prime}, k\right),
$$

for $j=2,3$, where the closed differential 1-form is given by

$$
W_{j}(x, t, k)=[U d x+V d t] \mu_{j}(x, t, k), \quad j=2,3 .
$$

For an additional eigenfunction $\mu_{1}(x, t, k)$ taking into account asymptotically periodic boundary data, let $\tilde{\psi}(t, k)$ be the solution of the background $t$-part of the Lax pair

$$
\tilde{\psi}_{t}+i f_{2}(k) \sigma_{3} \tilde{\psi}=\tilde{V} \tilde{\psi},
$$

where $\tilde{V}(t, k)$ is the matrix-valued function defined by replacing $u(0, t), u_{x}(0, t)$ and $u_{x x}(0, t)$ by $\tilde{g}_{0}(t), \tilde{g}_{1}(t)$ and $\tilde{g}_{2}(t)$, respectively, that is,

$$
\tilde{V}(t, k)=\left(\begin{array}{cc}
-2 i \lambda k \tilde{g}_{0}^{2} & H(k, t) \\
\lambda H(-k, t) & 2 i \lambda k \tilde{g}_{0}^{2}
\end{array}\right)
$$

where

$$
H(k, t)=2 \lambda \tilde{g}_{0}^{3}(t)+4 k^{2} \tilde{g}_{0}(t)+2 i k \tilde{g}_{1}(t)-\tilde{g}_{2}(t) .
$$

We seek a solution in the form of $\tilde{\psi}(t, k)=\mathscr{E}(t, k) e^{-i \Omega(k) t \sigma_{3}}$, where $\mathscr{E}(t, k)$ is a periodic function with period $\tau$ and $\Omega(k)$ is a complex-valued function. Then the solution $\mu_{1}(x, t, k)$ is defined by

$$
\begin{aligned}
\mu_{1}(x, t, k)= & e^{-i f_{1}(k) x \hat{\sigma}_{3}}\left\{\mathscr{E}(t, k)+\int_{0}^{x} e^{i f_{1}(k) x^{\prime} \hat{\sigma}_{3}}\left[U\left(x^{\prime}, t\right) \mu_{1}\left(x^{\prime} t, k\right)\right] d x^{\prime}\right. \\
& \left.-\mathscr{E}(t, k) \int_{t}^{\infty} e^{i \Omega(k)\left(t^{\prime}-t\right) \hat{\sigma}_{3}}\left[\mathscr{E}^{-1}\left(V_{0}-\tilde{V}\right)\left(t^{\prime}, k\right) \mu_{1}\left(0, t^{\prime}, k\right)\right] d t^{\prime}\right\},
\end{aligned}
$$


where $V_{0}(t, k)=V(0, t, k)$. The functions $\mathscr{E}(t, k)$ and $\Omega(k)$ can be defined as follows (see [19] for some details). Let $\psi(t, k)$ be the solution of the background $t$-part of the Lax pair (3.7) normalized by $\psi(0, k)=I$ and $M(k)=\psi(\tau, k)$ be the $2 \times 2$ monodromy matrix. The eigenvalues of $M(k)$ denoted by $\rho(k)$ and $\rho(k)^{-1}$ are given by

$$
\rho(k)=\frac{1}{2}(\operatorname{tr} M(k)-\sqrt{\Delta(k)}), \quad \Delta(k)=(\operatorname{tr} M(k))^{2}-4 .
$$

Let $\mathscr{P}$ be the set of branch points defined by $\mathscr{P}=\left\{k \in \mathbb{C} \mid \Delta(k)=0\right.$, or $M_{12}(k)=0$, or $\left.M_{21}(k)=0\right\}$ and $\mathscr{C}$ be a branch cut connecting all points in $\mathscr{P}$. From the Floquet theory it follows that letting the $2 \times 2$ matrix $\mathscr{B}(k)$ given by

$$
\mathscr{B}(k)=\frac{1}{\tau} \log M(k), \quad k \in \mathbb{C} \backslash \mathscr{C},
$$

the periodic function $\mathscr{E}(k)$ with period $\tau$ is defined by

$$
\mathscr{E}(k)=\psi(t, k) e^{-t \mathscr{B}(k)} \tilde{S}(k), \quad k \in \mathbb{C} \backslash \mathscr{C},
$$

where $\tilde{S}(k)$ is the matrix consisting of the eigenvectors corresponding the eigenvalues $\rho(k)$ and $\rho(k)^{-1}$ of $M(k)$ with $\operatorname{det} \tilde{S}(k)=1$. Then the function $\tilde{\psi}(t, k)$ in the form of

$$
\tilde{\psi}(t, k)=\mathscr{E}(t, k) e^{-i \Omega(k) t \sigma_{3}}, \quad k \in \mathbb{C} \backslash \mathscr{C},
$$

is the solution of (3.7), where $\Omega(k)$ is the eigenvalue of $i \mathscr{B}(k)$, that is,

$$
\Omega(k)=\frac{i}{\tau} \log \rho(k) .
$$

Furthermore, we introduce the domains $\left\{D_{j}\right\}_{1}^{4}$ given by

$$
\begin{array}{ll}
D_{1}=\left\{\operatorname{Im} f_{1}(k)>0, \operatorname{Im} \Omega(k)>0\right\}, & D_{2}=\left\{\operatorname{Im} f_{1}(k)>0, \operatorname{Im} \Omega(k)<0\right\}, \\
D_{3}=\left\{\operatorname{Im} f_{1}(k)<0, \operatorname{Im} \Omega(k)>0\right\}, & D_{4}=\left\{\operatorname{Im} f_{1}(k)<0, \operatorname{Im} \Omega(k)<0\right\} .
\end{array}
$$

\subsection{Perturbative expansions}

We expand the solution of (3.1) as a formal power series in small $\varepsilon>0$ :

$$
u(x, t)=\sum_{N=1}^{\infty} \varepsilon^{N} u^{(N)}(x, t) .
$$

Substituting this expansion into (3.1), at each order in the perturbative expansion, we find

$$
\begin{aligned}
O(\varepsilon): & u_{t}^{(1)}+u_{x x x}^{(1)}=0, \\
O\left(\varepsilon^{2}\right): & u_{t}^{(2)}+u_{x x x}^{(2)}=0, \\
O\left(\varepsilon^{3}\right): & u_{t}^{(3)}+u_{x x x}^{(3)}-6 \lambda\left(u^{(1)}\right)^{2} u_{x}^{(1)}=0, \\
O\left(\varepsilon^{4}\right): & u_{t}^{(4)}+u_{x x x}^{(4)}-6 \lambda\left[\left(u^{(1)}\right)^{2} u_{x}^{(2)}+2 u^{(1)} u^{(2)} u_{x}^{(1)}\right]=0, \cdots, \\
O\left(\varepsilon^{N}\right): & u_{t}^{(N)}+u_{x x x}^{(N)}-6 \lambda\left\{u^{2} u_{x}\right\}_{N}=0, \cdots,
\end{aligned}
$$


G. Hwang / The modified Korteweg-de Vries equation on the quarter plane with t-periodic data

where $\{\cdots\}_{N}$ denotes the coefficient of $\varepsilon^{N}$. We also expand the asymptotic boundary data for large $t$ in a formal power series in $\varepsilon$

$$
\tilde{g}_{j}(t)=\sum_{N=1}^{\infty} \varepsilon^{N} \tilde{g}_{j}^{(N)}(t), \quad j=0,1,2
$$

We now discuss the Dirichlet-to-Neumann map for the unknown boundary data in the limit of large $t$ and small $\varepsilon>0$. Proposition 3.1 below is the $\mathrm{mKdV}$ analog of Theorem 3.2 presented in [18] and the proofs are similar.

Proposition 3.1. Assume $\left\{\tilde{g}_{0}^{(N)}(t), \tilde{g}_{1}^{(N)}(t), \tilde{g}_{2}^{(N)}(t)\right\}$ are smooth periodic functions with period $\tau=$ $2 \pi / \omega>0$ and $\left\{u^{(N)}\right\}_{1}^{\infty}$ is a perturbative solution for the $m K d V(3.1)$ such that for each $N \geq 1$, $u^{(N)}(\cdot, t)$ is bounded in $L^{1}[0, \infty)$ and

$$
g_{j}^{(N)}(t)-\tilde{g}_{j}^{(N)}(t)=O\left(t^{-7 / 2}\right), \quad t \rightarrow \infty, \quad j=0,1,2,
$$

where $g_{0}^{(N)}(t)=u^{(N)}(0, t), g_{1}^{(N)}(t)=u_{x}^{(N)}(0, t)$ and $g_{2}^{(N)}(t)=u_{x x}^{(N)}(0, t)$ for $N \geq 1$. Suppose that the function $\tilde{g}_{0}^{(N)}(t)$ has the following Fourier series

$$
\tilde{g}_{0}^{(N)}(t)=\sum_{n=-\infty}^{\infty} a_{n}^{(N)} e^{i n \omega t}, \quad N \geq 1 .
$$

Then the Fourier coefficients of

$$
\tilde{g}_{1}^{(N)}(t)=\sum_{n=-\infty}^{\infty} b_{n}^{(N)} e^{i n \omega t}, \quad \tilde{g}_{2}^{(N)}(t)=\sum_{n=-\infty}^{\infty} c_{n}^{(N)} e^{i n \omega t}, \quad N \geq 1,
$$

are given by

$$
\begin{aligned}
b_{n}^{(N)} & =\frac{1}{2 i \kappa_{n}(1-\alpha)}\left\{G_{n}\left(\kappa_{n}\right)-G_{n}\left(\alpha \kappa_{n}\right)+F_{n}\left(\kappa_{n}\right)-F_{n}\left(\alpha \kappa_{n}\right)\right\}_{N}-2 i \alpha^{2} \kappa_{n} a_{n}^{(N)}, \\
c_{n}^{(N)} & =\frac{1}{1-\alpha}\left\{\alpha G_{n}\left(\kappa_{n}\right)-G_{n}\left(\alpha \kappa_{n}\right)+\alpha F_{n}\left(\kappa_{n}\right)-F_{n}\left(\alpha \kappa_{n}\right)\right\}_{N}-4 \alpha \kappa_{n}^{2} a_{n}^{(N)},
\end{aligned}
$$

where $\alpha=e^{2 i \pi / 3}$,

$$
\begin{aligned}
\kappa_{n}= \begin{cases}\frac{1}{2} e^{i \pi / 3}(n \omega)^{1 / 3}, & n \geq 0, \\
\frac{1}{2}(-n \omega)^{1 / 3}, & n<0,\end{cases} \\
G_{n}(k)=2 \lambda^{2} \sum_{l, m, p, q=-\infty}^{\infty} a_{l} a_{m} a_{p} d_{q} d_{n-q-p-m-l}, \\
F_{n}(k)=\sum_{l, m=-\infty}^{\infty}\left[4 \lambda k^{2} a_{l} d_{m} d_{n-m-l}-2 i \lambda k b_{l} d_{m} d_{n-m-l}-\lambda c_{l} d_{m} d_{n-m-l}\right] \\
+\sum_{l, m=-\infty}^{\infty}\left[4 i \lambda k a_{l} a_{m} d_{n-m-l}-2 \lambda a_{l} a_{m} a_{n-m-l}\right]
\end{aligned}
$$


and

$$
\begin{array}{ll}
a_{n}=\sum_{N=1}^{\infty} \varepsilon^{N} a_{n}^{(N)}, & b_{n}=\sum_{N=1}^{\infty} \varepsilon^{N} b_{n}^{(N)} \\
c_{n}=\sum_{N=1}^{\infty} \varepsilon^{N} c_{n}^{(N)}, & d_{n}(k)=\sum_{N=1}^{\infty} \varepsilon^{N} d_{n}^{(N)}(k)
\end{array}
$$

with

$$
d_{n}^{(N)}(k)=\frac{1}{8 i k^{3}+i n \omega}\left(4 k^{2} a_{n}^{(N)}+2 i k b_{n}^{(N)}-c_{n}^{(N)}-\left\{G_{n}(k)+F_{n}(k)\right\}_{N}\right) .
$$

Proof. Let

$$
R(t, k)=\frac{\mu_{1}(0, t, k)_{12}}{\mu_{1}(0, t, k)_{22}}, \quad k \in D_{1} / \mathscr{C}, \quad \tilde{R}(t, k)=\frac{\mathscr{E}(t, k)_{12}}{\mathscr{E}(t, k)_{22}}
$$

Note that as $t \rightarrow \infty, R(t, k) \sim \tilde{R}(t, k)$ for $k \in \bar{D}_{1} / \mathscr{C}$. Since $\tilde{\psi}(t, k)=\mathscr{E}(t, k) e^{-i \Omega(k) t \sigma_{3}}$ solves the background $t$-part (3.7), the function $\tilde{R}(t, k)$ solves the following Ricatti equation

$$
\begin{aligned}
\tilde{R}_{t} & +\left(8 i k^{3}+4 i \lambda k \tilde{g}_{0}^{2}\right) \tilde{R}+\left(2 \lambda^{2} \tilde{g}_{0}^{3}+4 \lambda k^{2} \tilde{g}_{0}-2 i \lambda k \tilde{g}_{1}-\lambda \tilde{g}_{2}\right) \tilde{R}^{2} \\
& -\left(2 \lambda k \tilde{g}_{0}^{3}+4 k^{2} \tilde{g}_{0}+2 i k \tilde{g}_{1}-\tilde{g}_{2}\right)=0 .
\end{aligned}
$$

We also expand the functions $\tilde{g}_{0}(t), \tilde{g}_{1}(t), \tilde{g}_{2}(t)$ and $\tilde{R}(t, k)$ as Fourier series

$$
\begin{array}{ll}
\tilde{g}_{0}(t)=\sum_{n=-\infty}^{\infty} a_{n} e^{i n \omega t}, & \tilde{g}_{1}(t)=\sum_{n=-\infty}^{\infty} b_{n} e^{i n \omega t}, \\
\tilde{g}_{2}(t)=\sum_{n=-\infty}^{\infty} c_{n} e^{i n \omega t}, & \tilde{R}(t, k)=\sum_{n=-\infty}^{\infty} d_{n}(k) e^{i n \omega t} .
\end{array}
$$

Substituting the above expansions into (3.21), the resulting equation leads to

$$
\left(8 i k^{3}+i n \omega\right) d_{n}(k)+G_{n}(k)+F_{n}(k)-4 k^{2} a_{n}-2 i k b_{n}+c_{n}=0,
$$

for $k \in \bar{D}_{1} / \mathscr{C}$, where $G_{n}(k)$ and $F_{n}(k)$ are defined in (3.18) and (3.19).

Let $\psi(t, k)=\sum_{N=0}^{\infty} \varepsilon^{N} \psi^{(N)}(t, k)$. Using the expansions (3.11), we find at $O(1) \psi^{(0)}(t, k)=$ $e^{-8 i k^{3} t \sigma_{3}}$ and at $O(\varepsilon)$

$$
\psi^{(1)}(t, k)=e^{-8 i k^{3} t \sigma_{3}}\left(\begin{array}{cc}
0 & \int_{0}^{t} e^{16 i k^{3} t^{\prime}} \tilde{H}^{(1)}\left(t^{\prime}, k\right) d t^{\prime} \\
\lambda \int_{0}^{t} e^{-16 i k^{3} t^{\prime}} \tilde{H}^{(1)}\left(t^{\prime},-k\right) d t^{\prime} & 0
\end{array}\right),
$$

where

$$
\tilde{H}^{(1)}(t, k)=4 k^{2} \tilde{g}_{0}^{(1)}(t)+2 i k \tilde{g}_{1}^{(1)}(t)-\tilde{g}_{2}^{(1)}(t) .
$$

Let $M^{(N)}(k)=\psi^{(N)}(\tau, k)$ and $M(k)=\sum_{N=0}^{\infty} \varepsilon^{N} M^{(N)}(k)$. Then, as $\varepsilon \rightarrow 0$

$$
M(k)=e^{-8 i k^{3} \tau \sigma_{3}}+\varepsilon e^{-8 i k^{3} \tau \sigma_{3}}\left(\begin{array}{cc}
0 & \int_{0}^{\tau} e^{16 i k^{3} t} \tilde{H}^{(1)}(t, k) d t \\
\lambda \int_{0}^{\tau} e^{-16 i k^{3} t} \tilde{H}^{(1)}(t,-k) d t & 0
\end{array}\right)+O\left(\varepsilon^{2}\right) .
$$


Thus, as $\varepsilon \rightarrow 0, \Delta(k)=-4 \sin ^{2}\left(8 k^{3} \tau\right)+O\left(\varepsilon^{2}\right)$ and

$$
\sqrt{\Delta(k)}=2 i \sin \left(8 k^{3} \tau\right)+O\left(\frac{\varepsilon^{2}}{\sin \left(8 k^{3} \tau\right)}\right), \quad \rho(k)=e^{-8 i k^{3} \tau}+O\left(\frac{\varepsilon^{2}}{\sin \left(8 k^{3} \tau\right)}\right)
$$

which implies that as $\varepsilon \rightarrow 0$,

$$
\Omega(k)=8 k^{3}+O\left(\varepsilon^{2}\right), \quad \mathscr{E}(t, k)=I+O(\varepsilon) .
$$

Thus, we know that the domains $D_{1}$ and $D_{3}$ deduce to $D_{+, 1} \cup D_{+, 2}$ and $D_{+, 3}$ as $\varepsilon \rightarrow 0$, respectively. Moreover, we find

$$
e^{i \Omega(k) t}=e^{8 i k^{3} t}\left(1+\varepsilon^{2} i t \Omega^{(2)}(k)+O\left(\varepsilon^{3}\right)\right), \quad \varepsilon \rightarrow 0,
$$

where $\Omega(k)=\sum_{N=1}^{\infty} \varepsilon^{N} \Omega^{(N)}(k)$.

Hence, the remaining proof is similar to that presented in [18] (see also [19]) and it is now enough to derive the relevant formulas for the Fourier coefficients. Let $\tilde{R}(t, k)=\sum_{N=1}^{\infty} \varepsilon^{N} \tilde{R}^{(N)}(t, k)$ and

$$
\tilde{R}^{(N)}(t, k)=\sum_{n=-\infty}^{\infty} d_{n}^{(N)}(k) e^{i n \omega t}, \quad t \rightarrow \infty, \quad k \in \bar{D}_{+, 1} \cup \bar{D}_{+, 2}
$$

Note that for each $N \geq 1$ the function $\tilde{R}^{(N)}(t, k)$ solves the Ricatti equation (3.21) at order $O\left(\varepsilon^{N}\right)$, that is,

$$
\begin{aligned}
& \left\{\tilde{R}_{t}+\left(8 i k^{3}+4 i \lambda k \tilde{g}_{0}^{2}\right) \tilde{R}+\left(2 \lambda^{2} \tilde{g}_{0}^{3}+4 \lambda k^{2} \tilde{g}_{0}-2 i \lambda k \tilde{g}_{1}-\lambda \tilde{g}_{2}\right) \tilde{R}^{2}\right. \\
& \left.-\left(2 \lambda k \tilde{g}_{0}^{3}+4 k^{2} \tilde{g}_{0}+2 i k \tilde{g}_{1}-\tilde{g}_{2}\right)\right\}_{N}=0, \quad k \in \bar{D}_{+, 1} \cup \bar{D}_{+, 2}
\end{aligned}
$$

Thus, in terms of the Fourier coefficients, we find, for $k \in \bar{D}_{+, 1} \cup \bar{D}_{+, 2}$,

$$
\left\{\left(8 i k^{3}+i n \omega\right) d_{n}(k)+G_{n}(k)+F_{n}(k)-4 k^{2} a_{n}-2 i k b_{n}+c_{n}\right\}_{N}=0 .
$$

Since the transform $k \rightarrow \alpha k$ leaves $k^{3}$ invariant, where $\alpha=e^{2 i \pi / 3}$, we obtain the following additional equation for $k \in \bar{D}_{+, 1}$,

$$
\left\{\left(8 i k^{3}+i n \omega\right) d_{n}(\alpha k)+G_{n}(\alpha k)+F_{n}(\alpha k)-4 \alpha^{2} k^{2} a_{n}-2 i \alpha k b_{n}+c_{n}\right\}_{N}=0 .
$$

Note that the equation $8 i k^{3}+i n \omega=0$ has roots $k=\kappa_{n} \in D_{+, 1}$ given by (3.17). Evaluating at $k=\kappa_{n}$, we find the system

$$
\begin{aligned}
& \left\{G_{n}\left(\kappa_{n}\right)+F_{n}\left(\kappa_{n}\right)\right\}_{N}-4 \kappa_{n}^{2} a_{n}^{(N)}-2 i \kappa_{n} b_{n}^{(N)}+c_{n}^{(N)}=0, \\
& \left\{G_{n}\left(\alpha \kappa_{n}\right)+F_{n}\left(\alpha \kappa_{n}\right)\right\}_{N}-4 \alpha^{2} \kappa_{n}^{2} a_{n}^{(N)}-2 i \alpha \kappa_{n} b_{n}^{(N)}+c_{n}^{(N)}=0 .
\end{aligned}
$$

Solving the system (3.30) for $b_{n}^{(N)}$ and $c_{n}^{(N)}$, the Fourier coefficients $b_{n}^{(N)}$ and $c_{n}^{(N)}$ are given by (3.15) and (3.16), respectively. Finally, using (3.28) with (3.15) and (3.16), the Fourier coefficient $d_{n}^{(N)}(k)$ is given by (3.20c). 
Remark 3.1. When $N=1$, equations (3.15) and (3.16) yield

$$
b_{n}^{(1)}=-2 i \alpha^{2} \kappa_{n} a_{n}^{(1)}, \quad c_{n}^{(1)}=-4 \alpha \kappa_{n}^{2} a_{n}^{(1)},
$$

and substituting (3.31) into (3.20c) with $N=1$, we find

$$
d_{n}^{(1)}(k)=\frac{4 a_{n}^{(1)}}{8 i k^{3}+i n \omega}\left(k^{2}+\alpha^{2} \kappa_{n} k+\alpha \kappa_{n}^{2}\right)= \begin{cases}-\frac{i a_{n}^{(1)}}{2 k+\alpha(n \omega)^{1 / 3}}, & n \geq 0 \\ -\frac{i a_{n}^{(1)}}{2 k-\alpha^{2}(-n \omega)^{1 / 3}}, & n<0 .\end{cases}
$$

Similarly, when $N=2$, we find $b_{n}^{(2)}=-2 i \alpha^{2} \kappa_{n} a_{n}^{(2)}, c_{n}^{(2)}=-4 \alpha \kappa_{n}^{2} a_{n}^{(2)}$ and

$$
d_{n}^{(2)}(k)= \begin{cases}-\frac{i a_{n}^{(2)}}{2 k+\alpha(n \omega)^{1 / 3}}, & n \geq 0, \\ -\frac{i a_{n}^{(2)}}{2 k-\alpha^{2}(-n \omega)^{1 / 3}}, & n<0 .\end{cases}
$$

Note that the Fourier coefficients of the terms in the summations in $G_{n}(k)$ and $F_{n}(k)$ are always given in the known lower order terms. Thus, continuing this process, we can determine the Fourier coefficients $b_{n}^{(N)}, c_{n}^{(N)}$ and $d_{n}^{(N)}(k)$ for $N \geq 3$.

\section{Example}

As for an example, we consider $g_{0}(t)=\varepsilon \sin t$ for $t \geq 0$. In this case, $\omega=1$ and $a_{n}^{(N)}=0$ except for

$$
a_{1}^{(1)}=\frac{1}{2 i}, \quad a_{-1}^{(1)}=-\frac{1}{2 i} .
$$

From (3.31), it follows that $b_{n}^{(1)}=c_{n}^{(1)}=0$ possibly except for $b_{ \pm 1}^{(1)}$ and $c_{ \pm 1}^{(1)}$. Note that

$$
\kappa_{1}=\frac{1}{2} e^{i \pi / 3}, \quad \kappa_{-1}=\frac{1}{2}
$$

Thus, the Fourier coefficients $b_{ \pm 1}^{(1)}$ and $c_{ \pm 1}^{(1)}$ at $O(\varepsilon)$ are given by

$$
\begin{array}{ll}
b_{1}^{(1)}=-\alpha^{2} \kappa_{1}=\frac{1}{2} \alpha, & b_{-1}^{(1)}=\alpha^{2} \kappa_{-1}=\frac{1}{2} \alpha^{2}, \\
c_{1}^{(1)}=2 i \alpha \kappa_{1}^{2}=\frac{i}{2} \alpha^{2}, & c_{-1}^{(1)}=-2 i \alpha \kappa_{-1}^{2}=-\frac{i}{2} \alpha .
\end{array}
$$

Therefore, we find

$$
\tilde{g}_{1}^{(1)}=\frac{1}{2}\left(\alpha e^{i t}+\alpha^{2} e^{-i t}\right), \quad \tilde{g}_{2}^{(1)}=\frac{i}{2}\left(\alpha^{2} e^{i t}-\alpha e^{-i t}\right),
$$

which are exactly the same results found in [6]. Moreover, equation (3.32) implies that $d_{n}^{(1)}=0$ except for $d_{ \pm 1}^{(1)}$ :

$$
d_{1}^{(1)}(k)=-\frac{i a_{1}^{(1)}}{2 k+\alpha}=-\frac{1}{4 k+2 \alpha}, \quad d_{-1}^{(1)}(k)=-\frac{i a_{-1}^{(1)}}{2 k-\alpha^{2}}=\frac{1}{4 k-2 \alpha^{2}} .
$$

Note that when $N=2, b_{n}^{(2)}=c_{n}^{(2)}=d_{n}^{(2)}=0$. 
When $N=3$, equation (3.18) implies that $G_{n}^{(3)}(k)=0$, where we denote $G_{n}(k)=$ $\sum_{N=1}^{\infty} \varepsilon^{N} G_{n}^{(N)}(k)$ and $F_{n}(k)=\sum_{N=1}^{\infty} \varepsilon^{N} F_{n}^{(N)}(k)$. Also, we know that $b_{n}^{(3)}=c_{n}^{(3)}=0$ possibly except for $b_{ \pm 1}^{(3)}, b_{ \pm 3}^{(3)}, c_{ \pm 1}^{(3)}$ and $c_{ \pm 3}^{(3)}$. Note that

$$
b_{ \pm 1}^{(3)}=\frac{1}{2 i \kappa_{ \pm 1}(1-\alpha)}\left[F_{ \pm 1}^{(3)}\left(\kappa_{ \pm 1}\right)-F_{ \pm 1}^{(3)}\left(\alpha \kappa_{ \pm 1}\right)\right],
$$

where

$$
\begin{aligned}
F_{ \pm 1}^{(3)}(k)= & 4 i \lambda k\left(2 a_{\mp 1}^{(1)} d_{ \pm 1}^{(1)}+a_{ \pm 1}^{(1)} d_{\mp 1}^{(1)}\right) a_{ \pm 1}^{(1)}+2\left(4 \lambda k^{2} a_{ \pm 1}^{(1)}-2 i \lambda k b_{ \pm 1}^{(1)}-\lambda c_{ \pm 1}^{(1)}\right) d_{1}^{(1)} d_{-1}^{(1)} \\
& +\left(4 \lambda k^{2} a_{\mp 1}^{(1)}-2 i \lambda k b_{\mp 1}^{(1)}-\lambda c_{\mp 1}^{(1)}\right)\left(d_{ \pm 1}^{(1)}\right)^{2}-6 \lambda^{2} a_{\mp 1}^{(1)}\left(a_{ \pm 1}^{(1)}\right)^{2}
\end{aligned}
$$

Substituting the coefficients $a_{ \pm 1}^{(1)}, b_{ \pm 1}^{(1)}, c_{ \pm 1}^{(1)}$ and $d_{ \pm 1}^{(1)}(k)$ in (4.6), equation (4.5) yields

$$
b_{ \pm 1}^{(3)}=\frac{\lambda}{48}(3 \pm 5 i \sqrt{3}) \text {. }
$$

Similarly, note that

$$
b_{ \pm 3}^{(3)}=\frac{1}{2 i \kappa_{ \pm 3}(1-\alpha)}\left[F_{ \pm 3}^{(3)}\left(\kappa_{ \pm 3}\right)-F_{ \pm 3}^{(3)}\left(\alpha \kappa_{ \pm 3}\right)\right]
$$

where

$$
F_{ \pm 3}^{(3)}(k)=4 i \lambda k\left(a_{ \pm 1}^{(1)}\right)^{2} d_{ \pm 1}^{(1)}+\left(4 \lambda k^{2} a_{ \pm 1}^{(1)}-2 i \lambda k b_{ \pm 1}^{(1)}-\lambda c_{ \pm 1}^{(1)}\right)\left(d_{ \pm 1}^{(1)}\right)^{2}-2 \lambda\left(a_{ \pm 1}^{(1)}\right)^{3}
$$

and

$$
\kappa_{3}=\frac{3^{1 / 3}}{2} e^{i \pi / 3}, \quad \kappa_{-3}=\frac{3^{1 / 3}}{2} .
$$

Then we find the coefficients $b_{ \pm 3}^{(3)}$ given by

$$
b_{3}^{(3)}=\frac{\lambda \alpha^{2}}{32}\left(3-3^{1 / 3}\right), \quad b_{-3}^{(3)}=\frac{\lambda \alpha}{32}\left(3-3^{1 / 3}\right) .
$$

Thus, the Fourier series representation for $\tilde{g}_{1}(t)$ at $O\left(\varepsilon^{3}\right)$ is given by

$$
\begin{aligned}
\tilde{g}_{1}^{(3)}(t)= & \frac{\lambda \alpha^{2}}{32}\left(3-3^{1 / 3}\right) e^{3 i t}+\frac{\lambda \alpha}{32}\left(3-3^{1 / 3}\right) e^{-3 i t} \\
& +\frac{\lambda}{48}(3+5 i \sqrt{3}) e^{i t}+\frac{\lambda}{48}(3-5 i \sqrt{3}) e^{-i t} .
\end{aligned}
$$

In a similar way, the coefficients $c_{ \pm 1}^{(3)}$ and $c_{ \pm 3}^{(3)}$ can be computed as

$$
c_{ \pm 1}^{(3)}=-\frac{\lambda}{48}(\sqrt{3} \pm 33 i), \quad c_{ \pm 3}^{(3)}= \pm \frac{i \lambda}{32}\left(9-9^{1 / 3}\right)
$$

and then the Fourier series representation for $\tilde{g}_{2}(t)$ at $O\left(\varepsilon^{3}\right)$ is given by

$$
\begin{aligned}
\tilde{g}_{2}^{(3)}(t)= & \frac{i \lambda}{32}\left(9-9^{1 / 3}\right) e^{3 i t}-\frac{i \lambda}{32}\left(9-9^{1 / 3}\right) e^{-3 i t} \\
& -\frac{\lambda}{48}(\sqrt{3}+33 i) e^{i t}-\frac{\lambda}{48}(\sqrt{3}-33 i) e^{-i t} .
\end{aligned}
$$


It should be noted that in [6], the case of $\tilde{g}_{0}(t)=\varepsilon \sin t$ was considered and the similar results were derived. However, in this example, the coefficients of the terms $e^{ \pm i t}$ in (4.8) and (4.9) are explicitly determined and the coefficients of the terms $e^{ \pm 3 i t}$ verify the results found in [6].

\section{Acknowledgement}

The work is supported by the Daegu University Research Grant, 2015.

\section{References}

[1] A. S. Fokas, A unified transform method for solving linear and certain nonlinear PDEs, Proc. Roy. Soc. London A 453 (1997) 1411-1443.

[2] A. S. Fokas, On the integrability of certain linear and nonlinear partial differential equations, J. Math. Phys. 41 (2000) 4188-4237.

[3] A. S. Fokas, Integrable nonlinear evolution equations on the half-line, Comm. Math. Phys. 230 (2002) $1-39$.

[4] A. S. Fokas, A Unified Approach to Boundary Value Problems, (CBMS-NSF Regional Conference Series in Applied Mathematics) SIAM (2008).

[5] A. S. Fokas, The generalized Dirichlet-to-Neumann map for certain nonlinear evolution PDEs, Comm. Pure Appl. Math. LVIII (2005) 639-670.

[6] G. Hwang and A. S. Fokas, The modified Korteweg-de Vries equation on the half-line with a sine-wave as Dirichlet datum, J. Nonlinear Math. Phys. 20 (2013) 135-157.

[7] P. Deif, S. Venakides and X. Zhou, The collisionless shock region for the long time behavior of the solutions of the KdV equation, Commun. Pure Appl. Math. 47 (1994) 199-206.

[8] P. Deif, S. Venakides and X. Zhou, New results in small dispersion KdV by an extension of the steepest descent method for Riemann-Hilbert problems, Int. Math. Res. Notices 6 (1997) 286-299.

[9] P. Deift and X. Zhou, A steepest descent method for oscillatory Riemann-Hilbert problems, Bull. Amer. Math. Soc. 26 (1992) 119-123.

[10] P. Deift and X. Zhou, A steepest descent method for oscillatory Riemann-Hilbert problems. Asymptotics for the mKdV equation, Ann. Math. 137 (1993) 295-368.

[11] de Monvel A. Boutet, A. Its and V. Kotlyarov, Long-time asymptotics for the focusing NLS equation with time-periodic boundary condition, C. R. Math. Acad. Sci. Paris 345 (2007) 615-620.

[12] de Monvel A. Boutet, A. Its and V. Kotlyarov, Long-time asymptotics for the focusing NLS equation with time-periodic boundary condition on the half-line, Commun. Math. Phys. 290 (2009) 479-522.

[13] de Monvel A. Boutet, A. Kotlyarov, D. Shepelsky and C. Zheng, Initial boundary value problems for integrable systems: towards the long-time asymptotics, Nonlinearity 23 (2010) 2483.

[14] A. S. Fokas and J. Lenells, The unified method: I. Non-linearizable problems on the half-line, J. Phys. A: Math. Theor. 45 (2012) 195201.

[15] G. Hwang, The Fokas method: The Dirichlet to Neumann map for the sine-Gordon equation, Stud. Appl. Math. 132 (2014) 381-406.

[16] G. Hwang, A perturbative approach for the asymptotic evaluation of the Neumann value corresponding to the Dirichlet datum of a single periodic exponential for the NLS, J. Nonlinear Math. Phys. 21 (2014) 225-247.

[17] J. Lenells and A. S. Fokas, The unified method on the half-line: II. NLS on the half-line with $t$-periodic boundary conditions, J. Phys. A: Math. Theor. 45 (2012) 195202.

[18] J. Lenells and A. S. Fokas, The nonlinear Schrödinger equation with $t$-periodic data: II. Pertrbative results, Proc. R. Soc. A 471 (2015) 20140926.

[19] J. Lenells and A. S. Fokas, The nonlinear Schrödinger equation with $t$-periodic data: I. Exact results, Proc. R. Soc. A 471 (2015) 20140925. 